\title{
Nutritional Awareness for Denture Wearers
}

\author{
${ }^{1}$ Gayathri. J \\ Student- intern \\ Thai Moogambigai Dental College and Hospital, \\ Chennai, Tamil Nadu
}

\begin{abstract}
Proper nutrition is essential for the health of every individual. This includes the denture wearers' as well. Proper advice and knowledge must be given to the denture wearers by the dentist for maintaining the essential health of the individuals.
\end{abstract}

\section{Observation}

The relationship between the oral health and the overall general health of the body is complex and controversial in denture wearers. It is observed that the inability to chew in denture wearers is directly related to their oral health which influences their selection of diet which later affects the nutritional status of them.

\section{INTRODUCTION}

The key factors for successful aging are described as intact social networks, physical, mental and cognitive functionality. Food selection is influenced not only on individual preferences but also habits formed in childhood, family/regional culture and economic status. Restrictions in selecting certain foods among denture wearing patients affects the overall health as well as the oral health of the individual

\begin{tabular}{|c|c|}
\hline NUTRIENT & QUANTITY PER DAY \\
\hline Energy & $2000 \mathrm{Kcal}$ \\
\hline Protein & $50 \mathrm{~g}$ \\
\hline Fat & $70 \mathrm{~g}$ \\
\hline Saturated fatty acids & $24 \mathrm{~g}$ \\
\hline Carbohydrates & $310 \mathrm{~g}$ \\
\hline Sugar & $90 \mathrm{~g}$ \\
\hline Dietary fibre & $30 \mathrm{~g}$ \\
\hline Sodium(salt) & $2.3 \mathrm{~g}$ \\
\hline
\end{tabular}

Table 1

\section{NUTRITIONAL REQUIREMENTS FOR DENTURE WEARERS:}

Nutrition is defined as the prescribed levels of nutritional values that are mandatory in overall well-being of human beings in general.

The normal energy requirements among healthy adults are $2000 \mathrm{Kcalories}$.A normal adult requires minimum 2 to 2.5 litres of water per day. It is found that people who had less than 20 teeth did have poorer nutritional intake than those who had more teeth, regardless of denture use .This included;

- Protein
${ }^{2}$ Sameera Ashiqa Sathik

Student- intern

Thai Moogambigai Dental College and Hospital, Chennai, Tamil Nadu

- Polyunsaturated fats

- Fibre

- Eight vitamins (such as vitamin D and various B vitamins )

- Two minerals (calcium andzinc)

In case of older population, they prefer soft diets due to the difficulty in chewing and other geriatric factors.

\section{SYMPTOMS ASSOCIATED WITH VITAMIN DEFICIENCY}

- Brittle hair and nails (vitamin B)

- Mouth ulcers or cracks in the mouth

- Bleeding gums (vitamin C)

- Poor night vision and white growths on the eyes (vitamin A)

- Scaly patches and dandruff

- Hair loss (niacin ,biotin)

- Red or white bumps on the skin (vitamin A\&C) SYMPTOMS ASSOCIATED WITH PROTEIN DEFICIENCY

- Edema

- Fatty liver disease

- Skin hair and nail problems

- Loss of muscle mass

- Brittle bones

- Increased severity of infections

- Greater appetite and calorieintake.

\section{DIET COUNSELLING OF PATIENTS UNDERGOING PROSTHODONTIC TREATMENT}

The following factors are responsible for the causes of malnutrition or undernutrition especially in older patients:

1. Low economic status and lack of knowledge about nutritional importance

2. Acute or chronic illness which results in physical impairment

3. Old dentures or under maintained dentures/prosthesis leads an individual to exclude certain food items which are difficult to chew that has a major effect on proper nutritional intake

4. Mental health disorders such as depression, dementia, social isolation, loneliness and anxiety may affect their food intake 
5. Appetite loss due to multiple medication . The main objective of diet counselling for patients undergoing prosthodontic care is to correct imbalances in nutrient intake that interfere with body and oral health.

The quality of a denture wearing patients' diet can be influenced positively by nutrition counselling. Dietary questions can be incorporated into medical history from or presented in one of the readily available nutrition questionnaires administered at the first appointment Seeking help from a dietitian is also advisable .

For socially isolated or disabled older adults, there are community- based nutrition programmes including food stamps, home-delivery meals and communal programs served in local senior centers, churches, or community centers. These nutrition service can have a severe impact on meeting the daily nutrient intake and nutritional status of participating older adults .Dental providers can refer these patients to these programs. For patients who are crippled by poor chewing function and who are at the risk of compromised nutrition, commercially available liquid supplements exists that provide for adequate intake of proteins, minerals and calories.

\section{NUTRITION GUIDELINES FOR PATIENTS UNDERGOING PROSTHETIC TREATMENT}

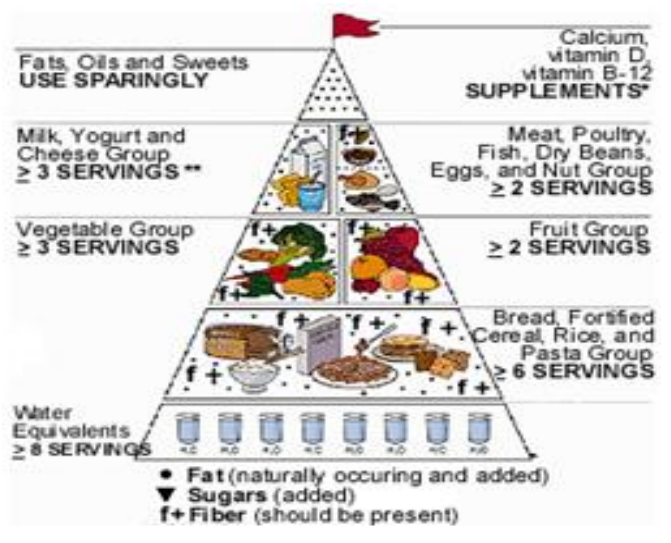

Fig 1

\section{THE FIVE FOOD GROUPS}

All the nutrients are required to maintain the overall health of an individual. This could be obtained by consuming a variety of foods in adequate quantity from the following five food groups :

1) Four servings of vegetables and fruits, subdivided into three categories : a)Two servings of good sources of vitamin C, this includes citrus fruits, salad greens and raw cabbage.

b) One serving of good source of pro vitamin A, this might be seen in deep green and yellow vegetables

c) One serving of potatoes and other vegetables and fruits.

2) Four servings of enriched breads, cereals and flour products.
3) Two servings of milk and milk-based foods.

4) Two servings of meats, fish, poultry, eggs, dried beans, peas, nuts.

5) Additional miscellaneous foods including fats, oils and sugars, the only serving recommendation is for about 2-4 table spoons of polyunsaturated fats, which supply essential fatty acids.

\section{CONCLUSION}

The above referred articles reveal that the majority of denture wearers are malnourished. This may be due to poor knowledge about the importance of nutrition among the denture wearers. Malnutrition among the denture wearing population could be avoided by their respective dentists and also nutritionist by proper guidance.

Denture wearers may be particularly vulnerable to both oral-systemic conditions and compromised nutritional health. The later can be addressed relatively readily with a professional dietician's help and dietary guidance should be an integral part of overall management of denture wearers. On the other hand, most oral-systemic conditions are very difficult and challenging to manage and may require alternative treatment interventions such as osseointergrated implant prosthesis.

\section{REFERENCES}

[1]. Snehiham A, Steele J G, Mascemes W, Taskos G. Prevalence's of implants of dental and oral disorders and their effects on eating among older people

[2]. Mullerk, Morasis J, Nutritional and anthropometric analysis of edentulous patients wearing implant over dentures.

[3]. Savoca M, Leng X. Severe tooth loss in older adults as a key indicator of compromised dietary quality.

[4]. Leight S.B. The application of a vulnerable populations conceptual model to rural health . Public Health Nurs.2003;20;440-8

[5]. Sahyoun NR, Lin CL, Krall E. Nutritional status of older adult is associated with dentition status.

[6]. Shinkai RS, Hatch JP, Sakai S, Mobley CC. Oral function and diet quality in a community-based sample.

[7]. Allison RA, Manski RJ. The supply of dentists and access to care in rural areas.

[8]. Hung HC, Joshipura KJ, Colditiz, MansonJE, RimEB, SpeizerFE. The association between tooth loss and coronary heart disease in men and women

[9]. Joshipura K J, Willet W C, DoughglassC. The impact of edentulousness on food and nutrient intake

[10]. Kiyak H A , Richmuth M.Barriers to and enables of older adult'suse of dental services 